Arch. Tierz., Dummerstorf 43 (2000) 3, 299-309

Aus dem Institut fur Tierzucht und Tierhaltung mit Tierklinik der Martin-Luther-Universitat Halle-Wittenberg

NORBERT MIELENZ, ELKE DITTMAR und LUTZ SCHÜLER

\title{
Effektivität der Zuchtwertschätzung mit transformierten Daten, ibberprüft mit Mutter-Töchter-Paaren eines Wachtelexperimentes
}

\author{
Herrn Professor Dr. D. Simon zum 70. Geburtstag gewidmet
}

\begin{abstract}
Summary
Title of the paper: Effectiveness of genetic evaluation with transformed data by using dam-daughter pairs of Japanese quails

In estimation of variance components with REML it has been assumed that the data were normally distributed. Egg production traits of poultry have been shown to exhibit markedly non-normal distributions. In this study, six traits from an unselected quail line were analysed. The original data were transformed using the wellestablished power transformation to approach normality. The genetic evaluation was carried out with a multipletrait animal model, based on transformed and untransformed data, respectively. Two traits of laying performance, of egg weight and body weight were analysed simultaneously. To compare the efficiency of breeding values the method of simulated selection with biological data (dam-daughter pairs) was used. To select the dams with intensities between $10 \%$ and $90 \%$ we used individual records and BLUP-breeding values, estimated with transformed and untransformed data. The response of such a selection was estimated using the corresponding daughter records. Only for the trait laying performance up to 200 days of life we could indicate an advantage of the transformation. It was shown, that by changing from one trait to multiple-trait genetic evaluation non-normality could be compensate. For $10 \%$ intensity the selection for individual laying performance provided an unexpected high response in comparing with the BLUP-method.
\end{abstract}

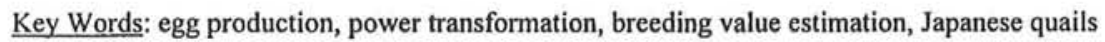

\section{Zusammenfassung}

Die Schätzung von Varianzkomponenten mit der REML-Methode geht von der Annahme aus, daß Normalverteilung für die Beobachtungen vorliegt. Typische asymmetrische Verteilungen besitzen die Merkmale der Eiproduktion beim Geflugel. Deshalb versucht man insbesondere die Legeleistung mit Hilfe einer Potenztransformation einer Normalverteilung anzunăhern. In dieser Arbeit wurde untersucht, ob durch Zuchtwertschätzung mit einem Mehrmerkmalsmodell, basierend auf transformierten Daten im Vergleich zur Verwendung von untransformierten Daten, eine Erhöhung des Selektionserfolges zu erwarten ist. Dazu wurde die Methode der simulierten Selektion mit biologischen Daten in Form von Mutter-Töchter-Paaren einer unselektierten Wachtelpopulation herangezogen. Die Auswahl der Mutter erfolgte mit Remontierungen von 10\% bis $90 \%$ nach vorkorrigierten Eigenleistungen und nach BLUP-Zuchtwerten, resultierend sowohl aus untransformierten als auch aus transformierten Daten. Der Selektionserfolg wurde als Differenz des Töchtermittels der selektierten Mütter zum Töchtermittel aller Müter geschătzt. Jeweils 2 Merkmale der Legeleistung, der Einzeleimasse und der Korpermasse gingen in die Zuchtwertschätzung ein. Lediglich für die Legeleistung nach 200 Lebenstagen konnte ein positiver Effekt der durchgefuhrten Transformation bestätigt werden. Es zeigte sich, daß Abweichungen eines Einzelmerkmals von der Normalverteilung durch Öbergang zu einer Zuchtwertschätzung mit Mehrmerkmalsmodellen teilweise kompensiert werden können. Für die scharfe Remontierung von $10 \%$ zeigte die Selektion nach Eigenleistung im Merkmal Eizahl gegentiber der Selektion nach BLUP-Zuchtwerten ein unerwartet gutes Abschneiden.

Schlusselwörter: Eiproduktion, Potenztransformation, Zuchtwertschătzung, Wachtel 
1. Einleitung

In der praktischen Zuchtarbeit ist die Varianzkomponentenschätzung (VKS) mit der REML-Methode unter Verwendung eines Tiermodells für viele Nutztierarten zum Standard geworden. Diese Methode garantiert gewisse optimale Eigenschaften, falls Normalverteilung vorliegt und bei Anwendung eines linearen gemischten Modells Linearität und Additivität für die festen Effekte sowie Varianzhomogenität für die zufälligen Resteffekte gegeben ist. Merkmale mit typischer asymmetrischer Verteilung sind insbesondere beim Geflügel anzutreffen. So besitzen die Merkmale Eizahl pro eingestellter oder überlebender Henne eine rechtssteile (linksschiefe) Verteilung. Dagegen weist das Merkmal Alter beim ersten Ei eine linkssteile (rechtsschiefe) Verteilung auf (vgl. IBE und HILL, 1988). Folgerichtig existieren Arbeiten, in denen vor der VKS mit der REML-Methode für das Merkmal Legeleistung eine Transformation auf Normalverteilung durchgeführt wurde (BESBES et al., 1991; WEI und VAN der WERF, 1993). Bei Merkmalen der Eiproduktion hat sich eine Variante der Potenztransformation (BOX und COX, 1964) durchgesetzt. So konnten IBE und HILL (1988) an Legehennendaten aus 6 Reinzuchtlinien nachweisen, daß transformierte Daten eine verbesserte Linearität zwischen Halbgeschwistermitteln und Eigenleistungen hervorbringen und im allgemeinen höhere Heritabilitäten als nichttransformierte Daten aufweisen. KOERHUIS (1996) analysierte die Eiproduktionsdaten aus 8 Generationen einer Hennenlinie vom Fleischtyp. Mit Hilfe der Box-Cox-Transformation konnte insbesondere die Linearität der Eltern-Nachkommen-Regression für die Merkmale Alter beim ersten Ei, Anzahl gelegte Eier nach 48 Wochen und für die prozentuale tägliche Eiproduktion erheblich gesteigert werden.

Die Wirksamkeit der Zuchtwertschätzung (ZWS) mit transformierten Daten wurde von SAVAS (1998) an Daten kommerzieller Legehennenlinien untersucht. Die ZWS mit einem Einmerkmals-Tiermodell, sowohl für transformierte als auch für untransformierte Daten, wurde für die akkumulierte Legeleistung von der 20 . bis 60 . Woche und für 4 Teillegeleistungen (20.-24. Woche, 25.-32. Woche, 33.-44. Woche und 45.60 . Woche) durchgeführt. Der Vergleich von Zuchtwerten, geschätzt mit untransformierten und Box-Cox-transformierten Daten, erfolgte mit Hilfe von Korrelationen und Übereinstimmungsraten in Abhängigkeit von verschiedenen Remontierungen (vgl. SAVAS et al., 1998). Für die Gesamtlegeleistung ergaben sich in den beiden untersuchten Linien Korrelationen von 0.96. Die Übereinstimmungsraten bei einer Remontierung von $10 \%, 30 \%$ bzw. $50 \%$ lagen z.B. in der ersten Linie bei $83 \%, 88 \%$ bzw. $93 \%$. Aus der einschlägigen Literatur resultiert die Ausage, daß durch Transformation die Linearität zwischen Selektionsdifferenz und Selektionserfolg erhöht wird und eine wirksamere Schätzung sowohl der Zuchtwerte als auch des Selektionserfolges mit transformierten Daten zu erwarten ist.

In dieser Arbeit wird obige Hypothese erstmals durch simulierte Selektion mit biologischen Daten in Form von Elter-Nachkommen-Paaren untersucht. Die Zuchtwertschätzung wird nicht auf Einmerkmalsfälle begrenzt, sondern wie in der Praxis üblich, mit Mehrmerkmalsmodellen bei gleichzeitiger Berücksichtigung von Legeleistungen, Eimassen und Körpermassen durchgeführt. Zusätzlich wird in den Vergleich die Selektion nach Eigenleistung, korrigiert um den Einfluß systematischer Umwelteffekte, einbezogen. 
2.

Material

Die Datenerhebungen erfolgten an Tieren einer unselektierten Wachtelpopulation (Zufallspaarung), die in drei Wiederholungen über 12 und einer Wiederholung über $6 \mathrm{Ge}$ nerationen im Verhältnis 1:2 (1 männliches Tier: 2 weibliche Tiere) verpaart wurden. Pro Generation sind 80 bis 120 weibliche und 40 bis 60 männliche Tiere zur Erzeugung der Folgegeneration verwendet worden. Der Versuch wurde in den Wiederholungen $\mathrm{A}, \mathrm{B}, \mathrm{C}$ und $\mathrm{D}$, die aus der gleichen Basispopulation stammen, realisiert. Eine ausfuhrliche Beschreibung von Tiermaterial, Haltungsbedingungen, Versuchsablauf und Paarungsstruktur findet man bei HEMPEL (1996). In Tabelle 1 sind für ausgewählte Merkmale die Leistungen der 4 Wiederholungen gemittelt über alle Generationen dargestellt.

Tabelle 1

Mittelwerte $(\bar{x})$ und Standardabweichungen (SD) in den 4 Wiederholungen gemittelt über alle Generationen (Means and standard deviations within the 4 repeats over all generations)

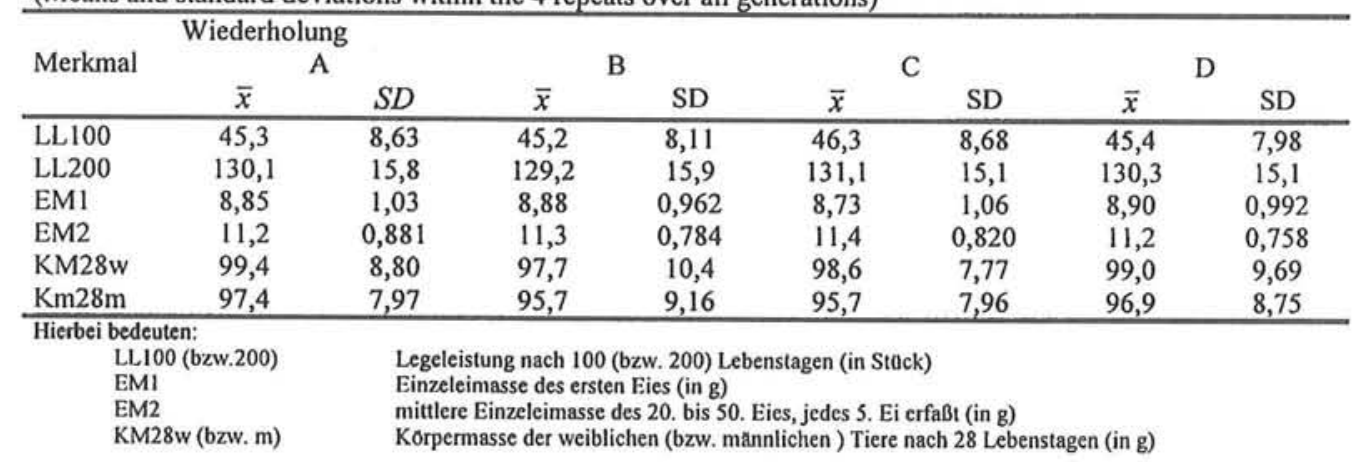

Die statistischen Maßzahlen in Tabelle 1 beruhen für Wiederholung A, B und D auf rund 1200 und für Wiederholung $\mathrm{C}$ auf rund 600 Beobachtungen. Zur Beschreibung der Verteilungen aller 6 Merkmale sind in Tabelle 2 unter anderem die Asymmetriemaße Schiefe und Exzeß für die Wiederholungen A und B aufgeführt. Die nachfolgenden Histogramme (Abb.) vermitteln einen Eindruck über die für Legeleistungen typische Form der Verteilung.
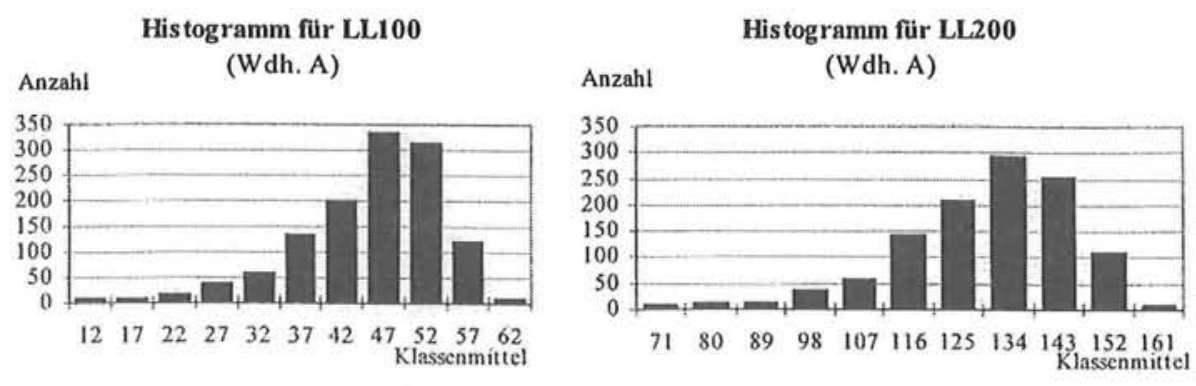

Abb.: Haufigkeitsverteilungen furr Merkmale der Legeleistungen (Frequency histograms for number of eggs) 
Aus Tabelle 2 geht hervor, daß die Merkmale LL100 und LL200 negative Schiefe und Medianwerte größer als der Mittelwert besitzen. Folglich ergibt sich eine typisch rechtssteile Verteilung, gemildert durch die hohen Minimalwerte von 10 bzw. 67 Eiern. Das Merkmal EM1 in den Wiederholungen A und B mit Werten von Schiefe und Exzeß nahe Null kann als normalverteilt angesehen werden.

Tabelle 2

Anzahl Beobachtungen (N), Minimum (min), Median sowie Schiefe $\left(\gamma_{1}\right)$ und Exzeß $\left(\gamma_{2}\right)$ fur Wiederholung A und B gemittelt über alle Generationen (Number of observations, minimum, median, skewness and kurtosis of repeat $\mathrm{A}$ and $\mathrm{B}$ calculated over all generations)

\begin{tabular}{|c|c|c|c|c|c|c|c|c|c|c|}
\hline \multirow[b]{2}{*}{ Merkmal } & \multirow[b]{2}{*}{$\mathrm{N}$} & \multicolumn{2}{|c|}{ Wiederholung } & \multicolumn{2}{|l|}{ A } & \multirow[b]{2}{*}{$\mathrm{N}$} & \multicolumn{2}{|c|}{ Wiederholung } & \multicolumn{2}{|l|}{ B } \\
\hline & & $\min$ & median & $\gamma_{1}$ & $\gamma_{2}$ & & $\min$ & median & $\gamma_{1}$ & $\gamma_{2}$ \\
\hline LL100 & 1237 & 10,0 & 47,0 & $-1,10$ & 1,48 & 1269 & 10,0 & 47,0 & $-0,959$ & 1,08 \\
\hline LL200 & 1138 & 67,0 & 133,0 & $-0,974$ & 1,23 & 1208 & 70,0 & 132,0 & $-0,837$ & 0,658 \\
\hline EM1 & 1237 & 6,12 & 8,82 & 0,505 & 1,45 & 1273 & 5,32 & 8,83 & 0,284 & 1,55 \\
\hline EM2 & 1236 & 8,35 & 11,2 & 0,036 & 0,048 & 1270 & 8,93 & 11,3 & 0,095 & 0,210 \\
\hline KM28w & 1315 & 63,0 & 100,1 & $-0,439$ & 0,715 & 1342 & 50,1 & 98,2 & $-0,438$ & 0,573 \\
\hline $\mathrm{KM} 28 \mathrm{~m}$ & 645 & 63,2 & 98,0 & $-0,435$ & 0,704 & 670 & 48,2 & 96,6 & $-0,661$ & 1,18 \\
\hline
\end{tabular}

3. Methode

3.1 Transformation der Daten

Die Transformation auf Normalverteilung erfolgte mit einer speziellen Variante der Potenztransformation, wie sie bereits von zahlreichen Autoren für Geflügeldaten benutzt wurde. Die von BOX und COX (1964) vorgeschlagene Transformation besitzt die Gestalt:

(1) $\quad z^{(t)}=\frac{y^{t}-1}{t \cdot\left(G_{y}\right)^{t-1}}$

Hierbei sind $z^{(t)}$ eine transformierte, standardisierte Beobachtung, $y>0$ eine untransformierte Originalbeobachtung, $G_{y}$ das geometrische Mittel der Ausgangsdaten sowie $t \neq 0$ der Parameter für die Potenz in der gesuchten Transformation. Von BOX und COX (1964) wurde gezeigt, wie der optimale Parameter $t$ mit Hilfe einer Varianzanalyse (VA) für Modell I ermittelt werden kann. Dazu sind die transformierten Daten für verschiedene Parameterwerte $t$ mit Modell I der VA auszuwerten, wobei neben den systematischen Umwelteffekten gewöhnlich auch die Vater- und Muttereffekte als fest angesehen werden müssen. Der Parameter $t$ wird so bestimmt, daß die zugehörige geschätzte Restvarianz minimal ausfällt. Tabelle 3 aus Abschnitt 4 enthält die berechneten $t$-Werte, wobei zusätzlich als Entscheidungshilfe die Asymmetriemaße Schiefe und Exzeß Berücksichtigung fanden.

3.2 Effizienzüberprüfung mit simulierter Selektion an biologischen Daten

Die Wirksamkeit der Zuchtwertschätzung (ZWS) mit transformierten und untransformierten Daten wurde anhand simulierter Selektion für die Merkmale LL100, LL200, EM1, EM2 und KM28w mit Mutter-Töchter bzw. für das Merkmal KM28m mit Mutter-Söhne-Paaren überprüft. Innerhalb der Wiederholungen ergaben sich bei Betraeh- 
tung zweier aufeinander folgender Generationen jeweils 80 bis 120 Mutter-Nachkommen-Paare. Die Paarbildung basierte auf dem von HEMPEL (1996) bereits auf feste Effekte korrigierten Datenmaterial. Zur ZWS der Mütter kam ein additives Tiermodell bei gleichzeitiger Einbeziehung aller 6 Merkmale bei Benutzung des Programmpaktes PEST (GROENEVELD und KOVACS, 1990) zum Einsatz. Die Schätzung der Populationsparameter, wie z.B. Heritabilitäten und genetische Korrelationen, erfolgte innerhalb der Wiederholungen mit den Daten aus allen verfügbaren Generationen unter Verwendung der REML-Methode bei Benutzung des Programmpaktes VCE (GROENEVELD, 1994; GROENEVELD und GARCIA-CORTES, 1998). Bei der Zuchtwertschätzung für die Mütter wurden nur Daten aus der Muttergeneration und aus weiter zurückliegenden Generationen herangezogen. Das heißt, die ZWS für Tiere auf Generation i $(0<1<$ n mit n-Anzahl der geprüften Generationen) benutzte nur Daten aus den Generationen 1 bis i. Zur Prüfung des Einflusses der Selektionsintensität auf die Schätzung des Selektionserfolges sind Remontierungen von $10 \%$ bis $90 \%$, gestaffelt in $10 \%$-Schritten verwendet worden. Die Selektionsdifferenz (SD) wurde berechnet als Abweichung des Mittels der Zuchtwerte selektierter Mütter vom Mittel der Zuchtwerte aller Mütter. Der Selektionserfolg (SE) für alle 6 untersuchten Merkmale wurde berechnet als Abweichung des Töchter- bzw. Söhnemittels der selektierten Mütter vom entsprechenden Nachkommenmittel aller Mütter. Durch Bildung des Mittelwertes über alle aufeinanderfolgenden Mutter-Nachkommen-Paare der 4 Wiederholungen wurde unter Beachtung der Anzahl Paare eine Schätzung für die SD und den zugehörigen SE in Abhängigkeit von der Remontierung $\alpha$ erhalten.

Betrachtet man alle 4 Wiederholungen, so ergeben sich insgesamt $(3 \times 11+5)=38$ aufeinanderfolgende Generationen mit Mutter-Nachkommen-Paaren, wobei die Anzahl der vorhandenen Paare variiert. Folglich stehen zur Schätzung des SE, der zu erwarten ist, wenn aus rund 80 bis 120 Tieren mit Remontierung $\alpha$ ausgewählt wird, $\mathrm{n}=38$ verschiedene Schätzwerte zur Verfügung. Die Schätzung des Selektionserfolges und seiner Varianz erfolgte unter Verwendung von Töchterleistungen bei Wichtung mit der Anzahl Elter-Nachkommen-Paare wie nachfolgend aufgeführt.

$$
\begin{array}{lll}
\bar{x}_{w}=S E=\frac{\sum_{i} w_{i} x_{i}}{\sum_{i} w_{i}} & \text { mit } & x_{i}=\bar{x}_{T_{i}}^{s}-\bar{x}_{T_{i}} \\
s_{x}^{2}=\frac{\sum_{i} w_{i}\left(x_{i}-\bar{x}_{w}\right)^{2}}{d} & \text { mit } & d=\sum_{i} w_{i}-\frac{\sum_{i} w_{i}^{2}}{\sum_{i} w_{i}}
\end{array}
$$

Hierbei sind:

$$
\begin{array}{ll}
\bar{x}_{T_{i}}^{s} \quad \text { Mittelwert der Töchter in Merkmal } x \text { von auf „Generation“ } i \text { selektierter Mütter } \\
\bar{x}_{T_{i}} \quad \text { Mittelwert der Töchter in Merkmal } x \text { von allen Müttern auf „Generation“ } i \\
w_{i} \quad \text { Anzahl der Mutter-Töchter-Paare gebildet mit Müttern aus „Generation“ } i
\end{array}
$$

Ist der Wichtungsfaktor $w_{i}$ gleich einer Konstanten für alle $i$, so ergeben sich aus (2) und (3) die bekannten Standardformeln für den Mittelwert und die Varianz aus $n \mathrm{Be}$ obachtungen. 
Die Zuchtwertschätzung der Mütter mit der BLUP-Methode unter Verwendung eines 6-Merkmalstiermodells erfolgte sowohl mit transformierten als auch untransformierten Daten. Alle Merkmale gingen transformiert ein, auch wenn der Parameter $\mathrm{t}$ mit Werten nahe Eins nur geringe Abweichungen von der Normalverteilung anzeigte. Für die Merkmale, in denen die Eigenleistung (EL) der Mutter vorlag, wurde der SE basierend auf der BLUP-Methode, dem SE, beruhend ausschließlich auf Selektion nach Eigenleistung, gegenübergestellt.

Neben dem Standardfehlern wurde für alle geschätzten Selektionserfolge ein Mittelwertvergleich für gepaarte Stichproben durchgeführt. Das heißt, für die Selektionserfolge, resultierend aus 2 verschiedenen Methoden, angewendet auf die Eltern-Nachkommen-Paare innerhalb aufeinander folgender Generationen wurde die Differenz gebildet. Somit ergeben sich für eine Remontierung 38 Schätzwerte für die Signifikanzprüfung dieser Differenz gegen Null.

\section{Ergebnisse}

In der folgenden Tabelle 3 sind die t-Werte und die Heritabilitäten für transformierte und untransformierte Daten, gemittelt über alle Wiederholungen, dargestellt.

\section{Tabelle 3}

Optimale Transformationsparameter $\mathrm{t}$ und Heritabilităten, geschătzt mit transformierten und untransformierten Daten (gemittelt uber alle Wiederholungen) (Optimal transformation parameter and heritabilities, estimated with transformed and untransformed data over all repeats)

\begin{tabular}{cllllll}
\hline \multicolumn{1}{c}{ Parameter } & LL100 & LL200 & KM28m & KM28w & EM1 & EM2 \\
\hline \multicolumn{1}{c}{ t } & 2,657 & 3,412 & 2,083 & 2,119 & 0,208 & 0,831 \\
$\hat{h}^{2}$ (untransformiert) & $\mathbf{0 , 1 5 3}$ & 0,199 & 0,333 & 0,327 & 0,207 & 0,554 \\
$\hat{h}^{2}$ (transformiert) & $\mathbf{0 , 1 7 4}$ & 0,202 & 0,336 & 0,338 & 0,211 & 0,543 \\
\hline
\end{tabular}

Für die Legeleistungen und Körpermassen lagen die berechneten t-Werte über Zwei und für die Einzeleimassen jeweils unter Eins. Nach IBE und HILL (1988) besitzen Daten mit $t \geq 5$ eine hohe Schiefe während Daten mit $t<2$ als annähernd normalverteilt eingestuft werden. In unserer Studie wurden alle Daten, unabhängig vom berechneten t-Wert, einer Transformation unterzogen. Die Effekte der Potenztransformation auf die geschätzten Heritabilitäten waren nur gering. Lediglich für das Merkmal LL100 mit dem höchsten t-Wert, ergab sich eine Erhöhung der Heritabilität beim Übergang zu transformierten Daten von 0,153 auf 0,174.

Die Ergebnisse der simulierten Zuchtwertschätzung unter Verwendung von ElterNachkommen-Paaren (ausführlich in Abschnitt 3.2 beschrieben) zeigen die Tabellen 5, 6 und 7. Die Rangordnung der $\alpha$-Prozent besten Mütter erfolgte einerseits nach BLUP-Zuchtwerten mit untransformierten $\left(\mathrm{ZW}_{\mathrm{u}}\right)$ und transformierten $\left(\mathrm{ZW}_{\mathrm{t}}\right)$ Daten sowie andererseits soweit vorhanden aufgrund der Eigenleistung der Mutter. Zwischen transformierten und untransformierten Eigenleistungen muß nicht unterschieden werden. Da die gewählte Transformation monoton ist, liefert die Selektion nach transformierten und untransformierten Eigenleistungen zwangsläufig übereinstimmende Rangierungen der Mütter und somit identische Selektionserfolge.

Der realisierte Selektionserfolg obiger Zuchtwertschätzung und seine Varianz wurden anhand der Töchterleistungen gemäß der Formeln (2) und (3) geschätzt. 
Die Schätzung von Selektionsdifferenz (SD) und zugehörigem Selektionserfolg (SE) erfolgte für 9 verschiedene Remontierungsraten. Somit ergaben sich 9 Punktepaare $\left(\mathrm{SD}_{\mathrm{i}}, \mathrm{SE}_{\mathrm{i}}\right)$, mit denen eine lineare Regression durchgefuht wurde. Die Bestimmtheitsmaße $\left(\mathrm{R}^{2}\right)$ dieser Regression in Abhängigkeit der Zuchtwertschätzung bei den Müttern enthält Tabelle 4.

\section{Tabelle 4}

Linearităt des Zusammenhangs zwischen SD und SE bei Selektion der Mütter nach Eigenleistung (EL) und nach BLUP-Zuchtwerten (ZW) (Linearity of response to selection and selection differential by selection on individual performance (EL) and breeding values (ZW))

\begin{tabular}{lccccc}
\hline \begin{tabular}{c} 
Bestimmtheitsmaß \\
\multicolumn{1}{c}{$\mathrm{R}^{2}$}
\end{tabular} & $\begin{array}{c}\text { Merkmal } \\
\text { LL100 }\end{array}$ & LL200 & EM1 & EM2 & KM28w \\
\hline Untransf. EL & 0,875 & 0,917 & 0,542 & 0,996 & 0,985 \\
Transf. EL & 0,904 & 0,951 & 0,522 & 0,996 & 0,986 \\
Untransf ZW & 0,965 & 0,958 & 0,749 & 0,984 & 0,979 \\
\hline
\end{tabular}

Die Ergebnisse der Tabellen 5 bis 7 gestatten:

(a) den Vergleich der Selektionserfolge resultierend aus der Zuchtwertschätzung mit untransformierten und transformierten Daten

(b) den Vergleich zwischen Selektion aufgrund der Eigenleistung und BLUP-Zuchtwerten

Hinsichtlich Punkt a) läßt sich keine generelle Überlegenheit der Zuchtwerte basierend auf transformierten Daten über die Summe aller 6 Merkmale feststellen. Lediglich für das Merkmal LL200 lieferte die durchgeführte Transformation für 7 von 9 Remontierungen eine Überlegenheit. Für Remontierungen von $30 \%$ und $80 \%$ zeigte der Test für gepaarte Stichproben eine signifikante Überlegenheit der transformierten ZWS an, allerdings nur wenn ein Signifikanzniveau von $10 \%$ unterstellt wurde.

Der Mittelwertvergleich für abhängige Stichproben lieferte für eine Irrtumswahrscheinlichkeit von 5\% nur für das Merkmal KM28w signifikante Unterschiede zwischen Selektion nach Eigenleistung und BLUP-Zuchtwerten bei Remontierungen von $30 \%$ bis $80 \%$.

Auffällig gemäß Punkt b) sind einerseits das günstige Abschneiden der Selektion nach Eigenleistung für beide Legeleistungen bei einer Remontierung von $10 \%$ und andererseits die unerwartet hohe Überlegenheit der BLUP-Zuchtwerte für das Merkmal EM1. Es sei bemerkt, daß bei einer Irrtumswahrscheinlichkeit von $10 \%$ die Selektion nach Eigenleistung im Merkmal LL200 der Selektion nach untransformierten BLUPZuchtwerten signifikant uberlegen ist.

Nachfolgend werden die einzelnen Merkmalskomplexe diskutiert.

Die Ergebnisse aus Tabelle 5 zeigen furr das Merkmal LL200, bis auf die Remontierungen von $40 \%$ und $90 \%$, höhere Selektionserfolge bei den Töchtern, falls die Selektion der Mütter nach transformierten Daten erfolgt. Dieses Ergebnis spricht für die Zweckmäßigkeit der mit $\mathrm{t}=3,4$ durchgeführten Potenztransformation. Für Remontierungen von $30 \%$ bis $90 \%$ ergibt sich die erwartete Überlegenheit der Selektion nach Zuchtwerten im Vergleich zur Selektion ausschließlich nach Eigenleistungen.

Die Selektionserfolge nach Eigenleistung im Merkmal LL100 für 10\% Remontierung mit 1.72 Eiern und im Merkmal LL200 für 10\% bzw. 20\% Remontierung mit 3,35 bzw. 2.40 Eiern scheinen stark überschätzt. Dies wird deutlich durch Betrachtung der 
Bestimmtheitsmaße einer linearen Regression der SE auf die SD (vgl. Tab. 4). Gerade die Schätzungen des SE bei Selektion der Mütter nach Eigenleistung mit $10 \% \mathrm{bzw}$. $20 \%$ Remontierung verursachen das relativ geringe $R^{2}$ von 0,875 im Vergleich $z u R^{2}$ von 0.965 bei der Regression der SE auf die SD für untransformierte Zuchtwerte.

Tabelle 5

Selektionserfolge, geschătzt an den Töchtern (in Klammern Standardfehler), für die Selektion nach Eigenleistung (EL) sowie nach Zuchtwerten, geschătzt mit untransformierten $\left(\mathrm{ZW}_{\mathrm{u}}\right)$ und transformierten $\left(\mathrm{ZW}_{1}\right)$ Daten (Selection response by using transformed and untransformed data)

\begin{tabular}{|c|c|c|c|c|c|c|c|c|c|c|c|c|}
\hline \multirow{3}{*}{$\begin{array}{c}\begin{array}{c}\alpha \\
\text { (in \%) }\end{array} \\
10\end{array}$} & \multirow{2}{*}{\multicolumn{2}{|c|}{$\begin{array}{c}\text { LL100 } \\
\text { EL }\end{array}$}} & \multicolumn{10}{|c|}{ LL200 } \\
\hline & & & \multicolumn{2}{|c|}{$\mathrm{ZW}_{\mathrm{u}}$} & \multicolumn{2}{|c|}{$\mathrm{ZW}_{\mathrm{t}}$} & \multicolumn{2}{|c|}{ EL } & \multicolumn{2}{|c|}{$\mathrm{ZW}_{\mathrm{u}}$} & \multicolumn{2}{|c|}{$\mathrm{ZW}_{1}$} \\
\hline & 1,72 & $(0,36)$ & 1,36 & $(0,39)$ & 1,13 & $(0,41)$ & $3,35^{\circ}$ & $(0,76)$ & $1,98^{b}$ & $(0,74)$ & 2,16 & $(0,71)$ \\
\hline 20 & 0,88 & $(0,22)$ & 1,21 & $(0,29)$ & 1,10 & $(0,28)$ & 2,40 & $(0,41)$ & 1,94 & $(0,56)$ & 2,11 & $(0,55)$ \\
\hline 30 & 0,81 & $(0,16)$ & 0,82 & $(0,20)$ & 0,66 & $(0,22)$ & 1,68 & $(0,34)$ & $1,76^{a}$ & $(0,44)$ & $2,09^{\circ}$ & $(0,46)$ \\
\hline 40 & 0,63 & $(0,13)$ & 0,69 & $(0,17)$ & 0,66 & $(0,17)$ & 1,42 & $(0,25)$ & 1,57 & $(0,35)$ & 1,50 & $(0,32)$ \\
\hline 50 & 0,58 & $(0,10)$ & 0,42 & $(0,14)$ & 0,39 & $(0,15)$ & 1,23 & $(0,24)$ & 1,25 & $(0,27)$ & 1,25 & $(0,27)$ \\
\hline 60 & 0,47 & $(0,11)$ & 0,47 & $(0,13)$ & 0,40 & $(0,13)$ & 0,77 & $(0,22)$ & 0,95 & $(0,24)$ & 1,02 & $(0,24)$ \\
\hline 70 & 0,27 & $(0,11)$ & 0,36 & $(0,10)$ & 0,39 & $(0,09)$ & 0,57 & $(0,19)$ & 0,78 & $(0,19)$ & 0,78 & $(0,19)$ \\
\hline 80 & 0,25 & $(0,08)$ & $0,21^{*}$ & $(0,07)$ & $0,27^{\circ}$ & $(0,07)$ & 0,34 & $(0,15)$ & $0,53^{2}$ & $(0,16)$ & $0,67^{\circ}$ & $(0,17)$ \\
\hline 90 & 0,08 & $(0,05)$ & $0,04^{\circ}$ & $(0,05)$ & $0,10^{6}$ & $(0,05)$ & 0,26 & $(0,12)$ & 0,40 & $(0,11)$ & 0,34 & $(0,11)$ \\
\hline
\end{tabular}

Signifikanzen zum Niveau von $10 \%$ mit verschiedenen Buchstaben gekennzeichnet

Tabelle 6

Selektionserfolge, geschätzt an den Töchtern (in Klammern Standardfehler), für die Selektion nach Eigenleistung (EL) sowie nach Zuchtwerten, geschătzt mit untransformierten $\left(\mathrm{ZW}_{\mathrm{u}}\right)$ und transformierten $\left(\mathrm{ZW}_{\mathrm{t}}\right)$ Daten (Selection response by using transformed and untransformed data)

\begin{tabular}{|c|c|c|c|c|c|c|c|c|c|c|c|c|}
\hline \multirow{3}{*}{$\frac{\begin{array}{c}\alpha \\
\text { (in } \%)\end{array}}{10}$} & \multirow{2}{*}{\multicolumn{2}{|c|}{$\begin{array}{c}\text { EM1 } \\
\text { EL } \\
\end{array}$}} & \multicolumn{10}{|c|}{ EM2 } \\
\hline & & & \multicolumn{2}{|c|}{$\mathrm{ZW}_{\mathrm{u}}$} & \multicolumn{2}{|c|}{$\mathrm{ZW}_{1}$} & \multicolumn{2}{|c|}{ EL } & \multicolumn{2}{|c|}{$\mathrm{ZW}_{\mathrm{u}}$} & \multicolumn{2}{|c|}{$\mathrm{ZW}_{\mathrm{t}}$} \\
\hline & 0,059 & $(0,060)$ & 0,099 & $(0,045)$ & 0,114 & $(0,047)$ & 0,362 & $(0,035)$ & 0,327 & $(0,041)$ & 0,341 & $(0,040)$ \\
\hline 20 & $0,063^{2}$ & $(0,035)$ & $0,140^{b}$ & $(0,031)$ & 0,129 & $(0,042)$ & 0,297 & $(0,025)$ & 0,278 & $(0,024)$ & 0,273 & $(0,026)$ \\
\hline 30 & $0,052^{\mathrm{A}}$ & $(0,029)$ & $0,106^{\mathrm{b}}$ & $(0,028)$ & 0,102 & $(0,028)$ & 0,237 & $(0,019)$ & $0,251^{*}$ & $(0,015)$ & $0,246^{b}$ & $(0,014)$ \\
\hline 40 & $0,054^{\prime \prime}$ & $(0,023)$ & 0,093 & $(0,020)$ & $0,099^{b}$ & $(0,020)$ & 0,205 & $(0,013)$ & 0,202 & $(0,013)$ & 0,199 & $(0,013)$ \\
\hline 50 & 0,061 & $(0,019)$ & 0,084 & $(0,015)$ & 0,082 & $(0,017)$ & $0,147^{\prime}$ & $(0,011)$ & $0,159^{\prime}$ & $(0,010)$ & $0,164^{b}$ & $(0,010)$ \\
\hline 60 & 0,055 & $(0,015)$ & 0,071 & $(0,014)$ & 0,075 & $(0,014)$ & 0,117 & $(0,009)$ & 0,125 & $(0,008)$ & 0,124 & $(0,009)$ \\
\hline 70 & 0,044 & $(0,011)$ & 0,045 & $(0,010)$ & 0,048 & $(0,009)$ & 0,094 & $(0,008)$ & 0,100 & $(0,008)$ & 0,098 & $(0,007)$ \\
\hline 80 & 0,032 & $(0,008)$ & 0,035 & $(0,007)$ & 0,039 & $(0,007)$ & 0,064 & $(0,006)$ & 0,060 & $(0,086)$ & 0,058 & $(0,006)$ \\
\hline 90 & 0,012 & $(0,005)$ & 0,018 & $(0,005)$ & 0,018 & $(0,005)$ & 0,026 & $(0,004)$ & 0,027 & $(0,005)$ & 0,028 & $(0,005)$ \\
\hline
\end{tabular}

Signifikanzen zum Niveau von $10 \%$ mit verschiedenen Buchstaben gekennzeichnet

Bezüglich der Merkmale EM1 und EM2 liegen wie in Tabelle 6 ausgewiesen die SE für transformierte und untransformierte Zuchtwerte über alle Remontierungen betrachtet in der gleichen Größenordnung. Augenscheinlich ist jedoch, daß die Selektion nach Eigenleistung für das Merkmal EM1 der Selektion nach Zuchtwerten stark unterlegen ist, während für das Merkmal EM2 annähernd Gleichwertigkeit erreicht wird. Ein Blick auf die Bestimmtheitsmaße der Tabelle 4 klärt die Situation auf. Für das Merkmal EM1 ist $\mathrm{R}^{2}$ bei Selektion nach Eigenleistung gleich 0,542 . Durch den Übergang zu geschätzten Zuchtwerten, welche ein komplexes Merkmal repräsentieren, wird für die lineare Regresssion der SE auf die SD für untransformierte Zuchtwerte mit $\mathrm{R}^{2}=0,749$ eine wesentlich bessere Linearität erreicht. Dieser lineare Zusammenhang liegt für das Merkmal EM2 mit $\mathrm{R}^{2}=0,996$ bereits vor. Diese Tatsache und die hohe Heritabilität von 0,554 für dieses Merkmal können als Erklärung für die 
Arch. Tierz. 43 (2000) 3

Tabelle 7

Selektionserfolge, geschătzt an den Töchtern bzw. Söhnen (in Klammern Standardfehler), für die

Selektion nach Eigenleistung (EL) sowie nach Zuchtwerten, geschătzt mit untransformierten $\left(\mathrm{ZW}_{\mathrm{u}}\right)$

und transformierten $\left(\mathrm{ZW}_{1}\right)$ Daten.(Selection response by using transformed and untransformed data)

\begin{tabular}{|c|c|c|c|c|c|c|c|c|c|c|}
\hline \multirow{3}{*}{$\begin{array}{c}\begin{array}{c}\alpha \\
(\text { in } \%)\end{array} \\
10\end{array}$} & \multirow{2}{*}{\multicolumn{2}{|c|}{$\begin{array}{c}\text { KM28w } \\
\text { EL }\end{array}$}} & \multirow{2}{*}{\multicolumn{2}{|c|}{$\mathrm{ZW}_{\mathrm{u}}$}} & \multicolumn{6}{|c|}{$\mathrm{KM} 28 \mathrm{~m}$} \\
\hline & & & & & & & & & & \\
\hline & 2,31 & $(0,45)$ & 2,70 & $(0,43)$ & 2,79 & $(0,46)$ & $2,18^{n}$ & $(0,49)$ & $2,54^{6}$ & $(0,48)$ \\
\hline 20 & 2,05 & $(0,31)$ & 2,32 & $(0,32)$ & 2,21 & $(0,31)$ & 1,69 & $(0,29)$ & 1,76 & $(0,29)$ \\
\hline 30 & 1,48 & $(0,22)$ & 1,50 & $(0,25)$ & 1,51 & $(0,25)$ & 1,30 & $(0,24)$ & 1,33 & $(0,23)$ \\
\hline 40 & $1,16^{a}$ & $(0,17)$ & $1,47^{b}$ & $(0,21)$ & 1,42 & $(0,21)$ & 1,17 & $(0,21)$ & 1,18 & $(0,20)$ \\
\hline 50 & $0,93^{a}$ & $(0,17)$ & 1,19 & $(0,17)$ & $1,23^{b}$ & $(0,18)$ & 1,08 & $(0,13)$ & 1,07 & $(0,14)$ \\
\hline 60 & $0,65^{\mathrm{a}}$ & $(0,13)$ & $1,06^{b}$ & $(0,14)$ & $1,06^{b}$ & $(0,13)$ & 0,92 & $(0,11)$ & 0,87 & $(0,12)$ \\
\hline 70 & $0,53^{\circ}$ & $(0,10)$ & $0,76^{b}$ & $(0,11)$ & $0,76^{b}$ & $(0,11)$ & 0,68 & $(0,09)$ & 0,72 & $(0,09)$ \\
\hline 80 & $0,35^{n}$ & $(0,08)$ & 0,46 & $(0,07)$ & $0,49^{b}$ & $(0,07)$ & 0,49 & $(0,08)$ & 0,51 & $(0,08)$ \\
\hline 90 & 0,14 & $(0,05)$ & 0,22 & $(0,05)$ & 0,23 & $(0,05)$ & 0,26 & $(0,06)$ & 0,23 & $(0,06)$ \\
\hline
\end{tabular}

Gleichwertigkeit der 3 Selektionsvarianten dienen.

Für die Körpermasse der weiblichen Tiere konnte über die Summe der untersuchten Remontierungen keine Über- bzw. Unterlegenheit der Selektion auf transformierte Zuchtwerte beobachtet werden. Das Merkmal KM28w besitzt mit 0,327 eine mittlere Heritabilität, die t-Werte liegen im Mittel bei 2,119 und die lineare Regression der SE auf die SD bei Selektion nach Eigenleistung weist mit 0.985 bereits ein sehr hohes Bestimmtheitsmaß auf.

Für die Körpermasse der männlichen Tiere nach 28 Tagen konnten nur Mutter-SöhnePaare gebildet werden. Das Merkmal KM28m besitzt mit 0,333 ein mittleres $h^{2}$. Die in den 4 Wiederholungen gefundenen t-Werte, welche im Mittel bei 2,083 lagen, lassen nur eine geringe Abweichung von einer Normalverteilung erkennen. Trotzdem ergab die Selektion nach transformierten Zuchtwerten, bis auf Remontierungen von $50 \%$, $60 \%$ und $90 \%$ eine generelle Überlegenheit.

Dies könnte im Vergleich zu dem Merkmal KM28m durch das Fehlen einer Eigenleistung bei der Zuchtwertschätzung verursacht sein.

\section{Schlußfolgerungen}

Betrachtet man die Selektionserfolge bei den Töchtern für transformierte und untransformierte Zuchtwerte, so wird über die Summe aller 6 untersuchten Merkmale kein eindeutiger Trend sichtbar.

Für das Merkmal Legeleistung nach 200 Tagen (LL200), welches mit einem mittleren t-Wert von 3,4 am stärksten von der Normalverteilung abweicht, scheint eine Transformation angeraten.

Für die übrigen Merkmale mit durchschnittlichen t-Werten zwischen 0,208 und 2,657 ist die Situation weniger überschaubar. Man sollte jedoch bedenken, daß in der praktischen Zuchtarbeit pro Tier mehrere Informationsquellen in verschiedenen Merkmalen für die Zuchtwertschätzung zur Verfügung stehen. Folglich kann der geschätzte Zuchtwert für ein Merkmal sehr wohl einer Normalverteilung nahe kommen, obwohl die phänotypische Merkmalsausprägung diese Hypothese nicht bestätigt.

Hier liegt auch der wesentliche Unterschied zu bisher durchgeführten Untersuchungen, 
die nicht normalverteilte Merkmale einzeln und nicht eingebunden in einen Merkmalskomplex betrachten.

Abweichungen eines Einzelmerkmals von der Normalverteilung können durch Übergang zu einer komplexen Zuchtwertschätzung teilweise oder vollständig kompensiert werden.

Für mittlere Remontierungen liefert die BLUP-Zuchtwertschätzung, d.h. insbesondere die Berücksichtigung aller verwandtschaftlichen Informationen, wie erwartet gegenüber der Selektion nach Eigenleistung höhere Selektionserfolge im Merkmal Legeleistung. Die BLUP-Methode oder auch die Indexselektion liefern im Vergleich zur Eigenleistung eine genauere Schätzung für den additiv genetischen Effekt eines Tieres. Dominanzeffekte, die anscheinend bei der Legeleistung wirken (vgl. WEI und VAN der WERF, 1993), lassen die Vorhersage des Selektionserfolges (bei geringen Tierzahlen) nur mit großen Fehlern behaftet zu. Für sehr scharfe Selektionsintensitäten wird die Vorhersage des SE im Merkmal Legeleistung aufgrund von Heritabilitäten, geschätzt mit additiven Tiermodellen, sehr unsicher.

Die auf feste Umwelteffekte korrigierten Eigenleistungen können als Schätzungen für den (gesamten) genetischen Effekt eines Tieres angesehen werden, während die BLUP-Zuchtwerte eine Vorhersage für den additiv genetischen Effekt eines Tieres darstellen.

Bevor eine Transformation zum Einsatz kommt, sollte geprüft werden, ob z. B. durch Eliminierung von Ausreißern oder durch Erfassung und Definition der Merkmale eine Verbesserung der Verteilungseigenschaften erreicht werden kann. So scheint es wenig sinnvoll von der kumulativen Legeleistung zur täglichen Eiproduktion, gemessen in Prozent, überzugehen. In diesem Zusammenhang stellt sich die Frage nach der Berücksichtigung von Tieren, die über eine volle Legeperiode nur wenige Eier gelegt haben.

\section{Literatur}

BESBES, B.; DUCROCQ, V.; TAVERNIER, A.; FOULleY, J.L.; BOICHARD, M.; BEAUMONT, C.; PORTAIS, M.:

Estimation of genetic parameters of egg production traits of laying hens by restricted maximum likelihood applied to a multiple-trait reduced animal model. 42nd Annual meeting of the EAAP, Berlin, Sept. (1991)

BOX, G.E.P.; COX, D.R.:

An analysis of transformations. J. R. Statis. Soc. B, 26 (1964), 211-252

GROENEVELD, E.; KOVAC, M.:

A generalized computing procedure for setting up and solving mixed linear models. J. Dairy Sci. 73 (1990), 513-531

GROENEVELD, E.:

VCE - a multivariate, multimodel REML (co)variance component estimation package. In: Fifth World Congress on Genetics Applied to Livestock Production. Guelph 22 (1994), 47-48

GROENEVELD, E.; GARCIA-CORTES, A.:

VCE4.0, a (co)variance component package for frequentists and BAYESIANS: In. Sixth World Congress on Genetics Applied to Livestock Production. Armindahl 27 (1998), 455-458

HEMPEL, S.:

Untersuchungen zur Asymmetrie genetischer Parameter an Leistungsmerkmalen einer unselektierten Wachtelpopulation. Martin-Luther-Universităt Halle-Wittenberg, Diss., 1996 
IBE, S.N.; HILL, W.G.:

Transformation of poultry egg production data to improve normality, homoscedasticity and linearity of genotypic regression. J. Anim. Breed. Genet. 105 (1988), 231-240

KOERHUIS, A.N.M.:

Non-normality of egg production distributions in poultry and the effects of outlier elimination and transformation on size and curvilinearity of heritability. Livestock Prod. Sci. 45 (1996), 69-85

WEI, M.; VAN DER WERF, J.H.J.:

Animal model estimation of additive and dominance variances in egg production traits of poultry. J. Animal Sci. 71 (1993), 57-65

SAVAS, T.; PREISINGER, R.; RÖHE, R.; THOMSEN, H.; KALM, E.:

The effect of the Box-Cox transformation on the estimation of breeding values for egg production. $6^{\text {th }}$ SAVAS, T.:

World Congress on Genetics Applied to Livestock Production, 24 (1998), 353

Untersuchungen zur Verbesserung der Zuchtwertschătzung für Legeleistung bei Legehennen. ChristianAlbrechts-Universitat, Kiel, Diss., 1998

Eingegangen: 20.05.1999

Akzeptiert: 23.11 .1999

Anschrif der Verfasser

Dr. NORBERT MIELENZ, ELKE DITTMAR, Uni.-Prof. Dr. habil. LUTZ SCHÜLER

Institut fur Tierzucht und Tierhaltung mit Tierklinik der

Martin-Luther-Universităt Halle-Wittenberg

Adam-Kuckhoff-Straße 35

D-06108 Halle

E-Mail: mielenz@landw.uni-halle.de 
Arch. Tierz., Dummerstorf 43 (2000) 3, 310

Buchbesprechung

\title{
Praktische Rinderfütterung
}

\author{
GUSTAV BURGSTALLER
}

5. vollkommen überarbeitete Auflage, 88 Seiten, 67 Abbildungen, 86 Tabellen, Landbuch Verlag, Hannover, 1999, ISBN 3-78420-571-2, 29,80 DM

In nunmehr 5. vollkommen uberarbeiteter Auflage ist dieser, der praktischen Rinderfutterung gewidmete Buchtitel, erschienen. Den Darlegungen zur Fütterung der Milchkühe, Aufzuchtkalber, weiblichen Jungrinder, Zuchtbullen, Mastkălber, Mastbullen, Mastochsen und Mastfärsen sowie zur Fütterung in der Mutterkuhhaltung, sind Ausfuhrungen zur Verdauung und zum Stoffwechsel der Wiederkăuer, zur Bewertung der Futtermittel und über Einflüsse auf den Futterverzehr vorangestellt. In diesen Kapiteln vermittelt der Autor in gestraffter und verständlicher Form den neuen Erkenntnisstand. Es finden hierbei die neuen Empfehlungen der Gesellschaft für Ernăhrungsphysiologie zur Futterbewertung umfassende Berücksichtigung. Diese sind durch Rechenbeispiele untersetzt. Vom Verfasser werden desweiteren Energieschätzmethoden für Kraftuttermittel und Grundfutter sachkundig kommentiert.

Einen breiten Raum nimmt das Kapitel zur Fütterung der Milchkühe in den verschiedenen Stadien der Laktation und in der Trockensteherzeit ein. Ausgehend von Richtzahlen und Empfehlungen zum Energie- und Năhrstoffbedarf sowie zu deren Versorgung, werden zahlreiche Rechenbeispiele unter Einbeziehung einer umfangreichen Palette von Grundfuttermitteln vorgestellt. Dabei finden die Anforderungen an die Grundfutterqualităt umfassend Berücksichtigung. Den Abschluß in diesem Kapitel bilden kurzgefasste aber dennoch informative Ausfuhrungen über Einflüsse der Futterung auf Zusammensetzung und Beschaffenheit der Milch sowie über die Vermeidung von Fruchtbarkeits- und Stoffwechselstörungen durch gezielte Futterungsmassnahmen.

Wenngleich die Futterung der anderen Rinderkategorien weniger Raum einnimmt, sind die Darlegungen ebenfalls wissenschaftlich fundiert und gut verständlich abgefasst. Dies wird $u$. a. durch Rationsbeispiele vorteilhaft unterstilitzt.

Relativ knapp fallen die Ausfuhrungen über Futterzusatzstoffe aus, zumal diese noch verstreut erfolgen. Mehr Beachtung hătten auch der Einfluss der Futterung auf die Schlachtkörperbeschaffenheit und die Fleischqualität sowie der Zusammenhang zwischen Futterung und Umwelt verdient.

Der vorliegende Buchtitel bietet vor allem den Beratungskräften in der tierischen Produktion und den Milchproduzenten wichtiges Informationsmaterial fur ihre Arbeit.

HEINZ JEROCH, Halle 